\title{
EL PENSAMIENTO EDUCATIVO EN TRES PROVINCIAS ANDINAS DE LA LIBERTAD*
}

\author{
EDUCATIONAL CONCEPTIONS IN THREE \\ ANDEAN PROVINCES OF LA LIBERTAD
}

Saniel E. Lozano Alvarado ${ }^{1}$

\section{RESUMEN}

En el presente ensayo presentamos un panorama sobre la producción intelectual de investigadores educacionales; por lo tanto, no se trata de estudios biográficos, sino de concepciones, aportes, planteamientos y experiencias volcadas en el libro. El estudio, en realidad, forma parte de una investigación mucho más amplia referida al pensamiento educativo producido y desarrollado en La Libertad. En esta oportunidad, el ámbito está delimitado por el quehacer intelectual producido en las provincias de Sánchez Carrión (Huamachuco), Santiago de Chuco y Otusco.

Palabras Clave: Pensamiento educativo, Producción intelectual, La Libertad.

\section{TRAYECTORIAS MAGISTERIALES}

\subsection{EDUCADORES HUAMACHUQUINOS}

Julio Galarreta González es un distinguido escritor, periodista e investigador, dedicado de manera infatigable a explorar y difundir la producción literaria, cultural y educativa, especialmente de su jurisdicción. En su vasta producción destacan sus trabajos de tema educativo: "Cultura y educación" (1961), "Abelardo Gamarra: educador peruano" (1974), "Pensamiento pedagógico de Abelardo Gamarra" (1974), "Huamachuco: escritores y maestros" (1974).

* Recibido: 5 de julio del 2013; aprobado: 6 de diciembre del 2013.

1 Editor asociado de "In Crescendo" y Director de "Rayuelo", revista oficial de la Asociación Peruana de Literatura Infantil y Juvenil (APLIJ). Profesor de la Universidad Privada Antenor Orrego, de Trujillo. 
El libro "Abelardo Gamarra: educador peruano" se inscribe en el conjunto de textos dedicados a explorar y exaltar la trascendental obra pedagógica del notable escritor y periodista huamachuquino. En este libro, Galarreta, luego de presentar una exhaustiva cronología, desarrolla en forma sucesiva los temas: El Perú y Abelardo Gamarra, Semblanza de un maestro, Valores educativos en la vida de Abelardo Gamarra, La doctrina educativa de Abelardo Gamarra y Abelardo Gamarra en la Educación peruana.

La importante obra se complementa con una exhaustiva relación de artículos pedagógicos publicados por Gamarra en el periódico fundado y dirigido por él: "La Integridad".

Realmente no se conoce un trabajo tan acucioso, tan analítico y detallado. Seguramente, el amor y la devoción por el maestro, así como la seria preocupación por la formación de nuestros niños y jóvenes y el destino de la patria, cuyas raíces hay que formarlas desde los comienzos de la vida, explican en gran medida la producción de estas obras.

De todo el conjunto temático del libro interesa fijar la atención en estos apartados:

Sobre el valor y el sentido de la educación, Gamarra -según su paisanoenalteció el significado de ésta, diciéndonos que es algo muy serio, pues nos educamos para ser buenos ciudadanos, buenos hombres o buenas mujeres, "no para piruetear, gastar plata y establecer una sociedad hueca, vacía y tan a la volástica". Es que el pensamiento de Gamarra se centraba fundamentalmente en educar antes que instruir.

Acerca de la educación de los peruanos alejados de los centros urbanos, González señala que el pensamiento de Gamarra se encuentra en su proyecto de origen parlamentario: "escuelas en las haciendas y profesores ambulantes, por el cual luchó, fervoroso y tesonero, sin éxito alguno debido a la incomprensión de unos y al propósito frustrante de otros. Gamarra propuso tales escuelas y tales profesores apoyándose en una realidad evidente, incontrastable: es absolutamente imposible llevar la educación institucionalizada, con escuelas sostenidas por el Estado, a los ahijaderos, ayllus, vaquerías, haciendas aisladas, chácaras, estancias, etc., donde vive gran número de niños de ambos sexos. Hasta allá irían esas escuelas y esos profesores”.

También el autor rastrea el pensamiento educativo de Gamarra con respecto a la formación de los niños. Entonces claramente señala: 
Respecto a los niños, por quienes Gamarra sintió siempre predilección, sostuvo que los primeros años de la vida no están destinados a la asistencia a las escuelas, oponiéndose a la tendencia contemporánea, cada más absorbente y excesiva, de imponer trabajos mentales a la infancia con detrimento del caudal de fuerzas que el organismo infantil demanda para su desarrollo. Lo natural es que en las primeras edades de la vida no se desarrolle el desmesurado afán de hacer estudiar a los niños. En otro artículo indicó que de tres a siete años de edad, en los niños debe cultivarse la sensibilidad, enseñándoles a sentir, a creer, y llevando a su corazón todo lo que pueda formar en ellos la delicadeza, por lo mismo que la niñez es la época del ejemplo”.

Reconoció la presencia vital del factor lúdico en el niño y recomendó su aprovechamiento inteligente dentro de la educación de su infancia. Decía que para los niños la vida no es otra cosa que un juego, y por jugar dejan alimentos, sueño, golosinas. Se opuso a usar los juegos de gimnasia por constituir éstos una inversión de las leyes de la naturaleza. Defendió con ardor y con fundamento científico-pedagógico los juegos al aire libre. Por eso pidió una y otra vez campos para los niños.(1974: 80).

Otro tema que analiza Galarreta en la obra de Abelardo Gamarra es la importancia del trabajo, que debe practicarse desde la familia y el hogar, continuarse en la escuela y ratificarse en la sociedad. Además insistió en la ligazón del trabajo con el canto, la lectura y el ejercicio físico:

Que en la escuela los cánticos, las lecturas y las enseñanzas objetivas se orienten a crear en el educando un concepto vivencial del trabajo y de la dignidad que él cimenta. Que se eduque para la vida, desarrollando las facultades físicas, intelectuales y morales del niño y contribuyendo a despertar en él el gusto y amor por el trabajo. (1974: 81).

La función de la familia en la educación de los hijos es fundamental en la doctrina educativa de Gamarra, según descubre Galarreta:

Los niños que son mal dirigidos en su hogar y no son debidamente atendidos en la escuela, más tarde no responderán a las aspiraciones de sus progenitores ni a los propósitos del Estado, alejándose cada día más del hombre ciudadano que la patrianecesita.

No cumplen su misión los padres de familia que, librando la suerte y el porvenir de sus hijos al esfuerzo solo del maestro, no participan en las complejas tareas de la educación, ni en aquellos actos públicos que debieran ser honrados y estimulados con la presencia de los padres de los educandos y de los representantes de las instituciones políticas, edilicias, sociales y culturales de cada lugar (p. 85). 
También ocupan lugar central en el pensamiento educativo de Abelardo Gamarra la educación de la mujer, así como la importancia y la valoración de las lenguas indígenas.

En su libro "Huamachuco escritores y maestros" (1986), luego de unas notas afectivas, telúricas y raigales, Galarreta González ofrece una semblanza profesional, cultural y pedagógica de ilustres intelectuales huamachuquinos empezando por Sánchez Carrión; luego continúa con Nicolás Rebaza, el autor de "Los anales del Departamento de La Libertad en la Guerra de la Independencia"; Abelardo Gamarra, de quien explora acuciosa y apasionadamente su vida; César Vallejo, cuya tierra natal, en la época del ilustre poeta pertenecía a la provincia de Huamachuco; el narrador indigenista Ciro Alegría; el educador y filósofo Julio Chiriboga, quien fue profesor secundario en los colegios "Modelo" y "Anglo-americano", así como Director de Estudios del Colegio Militar Leoncio Prado. En el nivel superior se inició en la Facultad de letras de la Universidad Nacional Mayor de San Marcos como profesor de Filosofía; en 1941 presidió la Comisión Organizadora del Instituto Pedagógico Nacional; y culminó su trayectoria como Rector de la Universidad Nacional de Trujillo.

Posteriormente, Galarreta se refiere a Alfredo Rebaza Acosta, escritor e investigador nato, autor de varios textos de historia, como "Historia universal", "Historia del Perú y del mundo", "Historia general de la cultura". También publicó el no menos inquietante e ilustrativo "El problema educativo peruano".

A continuación, con amplia y detallada información, Galarreta destaca la personalidad de varios escritores y educadores de su provincia, como Juan D. Cisneros Rodríguez, educador que se inició desde la modesta condición de Auxiliar, hasta ejercer los cargos de profesor de Geografía General y del Perú, Director del Colegio Nacional San Nicolás, Inspector de Enseñanza en las provincias de Huamachuco y Cajabamba. Posteriormente, luego de una injusta subrogación, pasó a ejercer la docencia en Acobamba (Tarma) y Barranco. También fue uno de los principales gestores de la formación de la Federación Nacional de Maestros del Perú (1931-33), en la que fue, primero, Secretario de Publicidad y después, Secretario General.

Toribio Guerra Vereau, quien con su título de Normalista, regresó a Huamachuco, en 1922, como Director de la Escuela Elemental. De allí pasó a Otuzco como Director de un centro escolar y Comisionado Escolar de la provincia. En 1927 retornó a Lima para estudiar Letras en la Universidad Nacional 
Mayor de San Marcos y se graduó como Doctor en Historia con una tesis sobre José Faustino Sánchez Carrión. Fue designado Inspector de Educación de las provincias piuranas de Sullana y Ayabaca. Su producción intelectual se registra en varias publicaciones periodísticas y eventuales, como "Integridad", "La Antorcha”, "Impetu”, "El Comercio", etc.

Reynaldo Solano Gamboa (1900-1950), "maestro por vocación y destino (...). Cada mañana escolar la iniciaba con las notas melódicas (acompañado de su violín) y los versos del himno del Colegio, inspiración de otro gran profesor y literato, Juan Espejo Asturrizaga”.

La obra educativa de Solano "estuvo orientada por dos preocupaciones fundamentales: educar al niño en contacto con la naturaleza y proyectar la escuela hacia la sociedad. La primera la cumplió usando creativamente el huerto escolar y los paseos campestres, los que tenían doble finalidad: que el alumno disfrutara deportivamente la naturaleza y que, al mismo tiempo, vivenciara el cariño a su terruño. La segunda le permitió trascender a la sociedad, haciendo que ésta aprecie y estimule la obra de los estudiantes, quienes se proyectaban a través de oportunas escenificaciones de la historia nacional, de la vida provinciana y de la creación dramática universal. Y es que Reynaldo Solano tenía vocación y habilidad para el teatro.

Edmundo Paredes Olazábal "uno de los sobrevivientes de una generación de hombres ilustres de la provincia de Huamachuco, cuya presencia en acciones edificantes y ejemplares de civismo se ha perennizado en la memoria colectiva de su tierra natal". Autoridad política y municipal intachable, fue también gran animador cultural y de notable acción social. Ejerció la docencia en el Colegio San Nicolás y en su academia particular, así como en otros centros educativos de la provincia.

Florencia de Mora. Se trató de una “mujer magnánima, dueña de acendrado altruismo y de solidaria actitud filantrópica. Aún no se le ha dado todo el valor y toda la significación al gesto humanista y justiciero". Parte de su vida y sobre todo la trascendencia de su obra aún sin valorar plenamente se puede apreciar en estas líneas:

De su infancia y de su adolescencia nada se sabe, pues aparece, en Trujillo, a los 20 años de su edad, casada con el español Juan de Sandoval y Guzmán. Su padre, casado en Trujillo con la española Ana de Valverde, tuvo hijos, pero "no dejó ninguno por línea recta, sino por la transversal”. Doña Florencia no tuvo descendencia y quedó viuda a los 40 años, al morir su esposo, el 4 de noviembre 
de 1581, declarándola única y universal heredera de las haciendas que había dejado no sólo en Huamachuco, sino también en Trujillo, Otuzco, Santiago de Chuco, Pallasca y Cajabamba. Doña Florencia falleció posiblemente en los primeros años del siglo XVII, pero habiendo testado en 1587, 1591 y 1596. Toda su fortuna la dejó como herencia para los indígenas compatriotas suyos, estableciendo las condiciones del usufructo. Nicolás Rebaza proporciona la relación de los fundos así como la suerte que habían corrido hasta la época en la que él redactó sus Anales, 1897.

(...) Entre las disposiciones testamentarias de doña Florencia de Mora recordaremos las siguientes: La hacienda Collambay la destinó al cultivo de algodón, el que serviría para fabricar tocuyo en los obrajes de la hacienda Sinsicap, con el que, a su vez, se confeccionarían camisas para los indígenas pobres de los siete pueblos de Huamachuco. Los productos de la hacienda El Hospital estaban dedicados a medicinas y alimentos para los indígenas del distrito de Santiago. Ya en la época republicana, por acuerdos congresales se dispuso que los productos de las haciendas El Hospital, Tulpo y Yamobamba contribuyeran al sostenimiento del Colegio Nacional San Nicolás que antes funcionaba como Aulas de Gramática Latina y Filosofía (pp. 52 y 53).

Juana Infantes Vera. Destacada investigadora, que fue profesora de la Cátedra de Botánica Sistemática, así como Asesora Técnica del Jardín Botánico de la Universidad Nacional Mayor de San Marcos. Además de artículos en periódicos y revistas, los resultados de sus investigaciones se publicaron en numerosos folletos, como: Estudio taxonómico, químico y fitográfico de 27 especies del género festusa; Monografía de las 11 especies del género cantua; Relación de las 64 plantas frutales del Perú; y Estudio taxonómico, histológico y etnobotánico de algunas plantes útiles del Perú.

\section{OTROS MAESTROS Y ESCRITORES HUAMACHUQUINOS}

También, en su mencionada obra, Galarreta González enfatiza en la importante labor cumplida por la maestra Bertha Peña de Sánchez; el escritor y militar otuzcano educado en Huamachuco, Lorenzo Morachimo Torres; el economista Luis Briceño Contreras; los conocidos e importantes maestros y poetas Eloy Hulero, autor del popular y ameno "Poncho habano"; Santiago Gastañaduí , hermano mayor del poeta Néstor, y el escritor Wilfredo Ledesma Llaury, infatigable impulsor de la revista semestral "Impetu”, que editó en 20 números. También fue autor de dos obras inéditas: "Monografía de la provincia de Sánchez Carrión” y “Costumbres y folklore de la provincia de Sánchez Carrión”. 


\subsection{VOZ Y PRESENCIA EDUCATIVA Y CULTURAL DE OTUSCO}

En su libro "Cuatro ensayos sobre la Región La Libertad" (2000), Efraín Orbegoso Rodríguez dedica un amplio espacio a comentar la labor de destacados intelectuales, artistas y pensadores de la provincia de Otusco que han contribuido al desarrollo de la educación y la cultura, especialmente en el ámbito regional, pero también con vastas proyecciones hacia otros espacios.

La relación elaborada por Orbegoso Rodríguez, que debe ser incompleta, pero que no deja de ser representativa, es la siguiente:

Luis José de Orbegozo y Moncada, militar que tuvo destacada actuación en las luchas por la independencia Nacional, que llegó a ser Presidente del Perú entre 1833 y 1836 . Su labor fue destaca de modo especial por el historiador Jorge Basadre: "La ayuda material y moral que, con otros personajes, prestó a San Martín fue invalorable (...) su elección significó una reacción contra el caudillaje militar y autoritario gamarrista.

Teófilo Vergel Carranza, natural del distrito de Salpo, quien en asociación con el periodista Raúl E. Haya, fundaron el histórico diario "La industria", de Trujillo, uno de los más importantes del país.

Carmelo Carranza Luján, exaltado por Manuel Rodríguez Romero como "pionero de la minería en La Libertad y ejemplo de trabajo y lucha como fiel heredero de las cualidades de sus padres".

Mariano Alcántara Morachimo, notable artista del dibujo e insuperable xilografista, quien tuvo entre sus amigos a los pintores José Sabogal, Julia Codesido y Camilo Blas; también ejerció el periodismo con Ciro Alegría y Antenor Orrego. Propietario de la Librería Divulgación, en el céntrico jirón Gamarra, de Trujillo, allí recibía a escritores, artistas plásticos, políticos y numerosas personalidades, como, aparte de los citados, José Eulogio Garrido, Luis Alberto Sánchez y otros importantes hombres públicos; el médico e investigador, profesor en las universidades norteamericanas de Temple Universiti, Pensilvania y Filadelfia.

Juan E. Armas, autor de la "Guía de Trujillo", publicada en 1935 con motivo de la celebración del Cuarto Centenario de la ciudad y su quehacer cultural también se plasmó en las revistas de la Universidad Nacional de Trujillo. Con ocasión de la coronación canónica de la Virgen de la Puerta por su Santidad, el Papa Pío XII, como "Patrona del Norte del Perú y Reina de la Paz Universal" (27-10-43), durante el Tercer Congreso Eucarístico de Trujillo publicó en "El Regional", órgano oficial de la Asociación Otuscana, un artículo en el 
que declaraba haber leído "El Acta en la que el pueblo de Otusco juró en la Plaza Pública la Independencia del Perú, el 22 de junio de 1821, documento que lamentablemente no copió para su publicación y se perdió en el Archivo Parroquial, con lo cual se causó grave daño a la historia de Otusco.

Manuel María Hurtado, uno de los intelectuales de renombre de la provincia. Cuando murió en 1924 dejó una gran biblioteca y archivo, "que su criado L. Mantilla vendía por kilos a los comerciantes".

El artesano zapatero, pensador socialista y poeta Teodoro Caseaux Loyola.

El músico Félix Hernández Ríos, gran compositor, que con don Pompilio Carbonell y don Lizandro Morgan fueron el trío de músicos y ebanistas más famosos de la provincia.

El médico naturista Pablo Lázaro Villacorta, estudioso predestinado, que desde muy joven se dedicó a investigar las plantas medicinales y se hizo herbolario. "Entre sus pacientes hubo humildes, ricos, profesionales e industriales peruanos y extranjeros; y cuando el Concejo Provincial acordó otorgarle medalla de oro y diploma de honor, los médicos se opusieron y no se la dieron. Entonces ocurrió que el pueblo de Trujillo en masa se volcó a las calles y le obsequió un plato de plata con esta leyenda: "Lo que el Concejo le quitó, el pueblo de lo dio".

Saniel Lozano Alvarado. También Sigifredo Orbegoso incluye en su estudio al autor de este artículo, cuyo contenido omitimos por razones obvias.

El mismo investigador evoca la personalidad y trayectoria magisterial de los siguientes maestros:

Zalatiel Peralta Sánchez, "maestro por vocación y convicción”, fundador de la Escuela Elemental de Varones y autor de la "Leyenda del culto a la Virgen de la Puerta".

María Herminia Armas Méndez, fundadora de la primera Escuela Elemental de Otusco, que dirigió durante 50 años, gran difusora de canciones escolares y populares "para que los niños amen lo suyo y formen un gran coro".

María Esther Arangurí Luján, directora del Centro Escolar 252 de Mujeres, virtuosa del piano y magnífica cantante.

Blanca García Acevedo, fundadora de la Biblioteca Municipal "para lo cual estudió en Lima y pidió a otuscanos y padres de familia, amigos y parientes: estantes, casilleros, mesas, sillas, libros, revistas y mapas. Como se convirtió en "Maestra amorosa", explicaba a los alumnos los temas, aclaraba las cosas que no entendían e "impuso una conducta de estudio para llegar al saber". "Al 
irse de la Dirección de la Biblioteca en los años 90 los estudiantes y el pueblo de Otusco, en general, le rindieran homenajes de reconocimiento. Pero nadie impidió que al irse alcaldes ignorantes dejaran que ese gran foco de cultura que honró a la ciudad de Otusco durante 30 años, entrara en vergonzosa decadencia.

Celia Rosa Espejo Carranza, que se inició en la docencia en la Escuela Elemental de Mujeres como "meritoria”, pasó a ser maestra y después Directora del Centro Escolar 252. Su hogar fue un lugar de reunión de intelectuales y músicos, donde se organizan tertulias poéticas y musicales.

Juana Peralta García, quien "tenía concepto elevado de la vida y del trabajo y su filosofía personal consistía en tomar todo en serio según su importancia y valor y no más allá, como en ver y sacar al aire lo alegre y gracioso de las cosas. En ella la alegría de vivir era tan natural y cristalina como el brotar del agua en un manantial andino".

María Josefa Rodríguez Zavaleta, una de las grandes maestras otuzcanas. "Alegre, haciendo maravillas de dibujos en la pizarra para que nuestra ignorancia capte mejor sus enseñanzas. Preguntándonos para ver si todos habíamos entendido la lección y, acercándose a los "más lentitos" para repetirles a ellos solos, la lección que no habían entendido. Esta última delicadeza muestra hasta qué punto ella entendió a cabalidad su difícil profesión y su alta calidad de Maestra”.

$\mathrm{Al}$ margen del tema docente, también el autor destaca la trayectoria de las siguientes personalidades:

Jorge Angulo Argomedo, jurista especialista en Derecho del Trabajo y autor de "Apuntes y recuerdos de mi comarca, del Perú y América".

El poeta Roque Calderón de los Ríos, quien de joven fue seminarista, pero cuyos estudios truncó por falta de medios económicos; también cultivó la poesía, muestra de la cual una prima suya "se la entregó a Enrique Linares en 1970, quien desde hacía dos años se la pedía para publicarla"; pero no se ha sabido nada más.

Félix Castillo Narváez, prestigioso médico, investigador científico y profesor en la Universidad Nacional Mayor de San Marcos.

El empresario Arístides Castro Gamboa, autor también de los libros: "El carbón antracita", "La economía industrial del Perú” y "Represas de los Andes", así como de los poemarios "Tesoro" e "ilusiones", cuya temática es el reconocimiento a la labor de campesinos y mineros.

El médico sinsicapino Wálter Cruz Vilca. 
El "guardia enfermero" Aníbal García Acevedo, activo militante de la Cruz Roja Peruana con reconocimiento internacional, especialmente en Ecuador, Colombia y Bucarest, donde fue elegido Miembro del Comité Consultivo de la Cruz Roja”.

El ingeniero Pedro José González Cueva, gran luchador social, que estudió las riquezas naturales de Salpo, Otusco y la Libertad, que soñó sin conseguirlo con la creación de la provincia de Salpo.

El magnífico y extraordinario escultor de la piedra Isidro Gutiérrez, natural del caserío de Cochaya, distrito de Salpo, artista de gran reconocimiento internacional.

El catedrático de la Universidad Nacional de Trujillo, Alfredo Gutiérrez Ibáñez, uno de los fundadores del Ateneo Popular de Trujillo, cuya labor cultural y de difusión fue singular hasta 1932.

El comerciante y transportista Angel Gutiérrez Sánchez.

El maestro y poeta de inspiración telúrica, Fidel Horna Cortijo.

El artista plástico de justo reconocimiento, Max Lois Lázaro Moya (“Urmo").

El militar y poeta Lorenzo Morachimo Torres, autor de importantes obras narrativas, especialmente de temática histórica, como por ejemplo: "Mi capote" y "Huasimo-Cazaderos", subtitulado "Un episodio de la campaña militar de 1941".

El físico nuclear salpino Modesto Montoya Zavaleta, de gran reconocimiento nacional e internacional.

El narrador humorista de inspiración popular y rural Jesús Euclides Santa María Corcuera

El pintor Armando Reyes Castro, natural de Agallpampa, un maestro del retrato, con varias exposiciones en el país y el extranjero. Fue miembro del Grupo "Trilce", de Trujillo.

El periodista Manuel Rodríguez Romero, ex Decano del Colegio de Periodistas de La Libertad.

El abogado y jurista Sigifredo Orbegoso Venegas, ex catedrático de la Universidad Nacional de Trujillo, actual rector de la Universidad César Vallejo, autor de varios libros especialmente de temática jurídica y política.

Eudoro Moreno Herrera, premiado por la FAO por sus méritos en el campo de la alimentación. 


\section{El educador Víctor Moya Méndez Acevedo.}

El abogado Héctor Ponce Arteaga, natural de Usquil.

En una sección especial del mismo trabajo, se incluye un artículo de Julio Galarreta González, titulado "Los Orbegozo de Otusco", en el que destaca los aportes de tres representantes de la intelectualidad otuscana: el poeta Ismael Orbegoso Polo, el geógrafo Efraín Orbegoso Rodríguez y el periodista Manuel Jesús Orbegozo.

Finalmente, la sección respectiva se cierra con el apartado: "Otuscanos: rectores y vicerrectores, en el que registra la trascendente labor en sus respectivos cargos al frente del Seminario San Carlos y San Marcelo, de Trujillo, de: Matías Martínez de Otiniano, José Vicente Martínez de Otiniano, Juan Antonio Luján, José Manuel Corcuera, José Manuel Corcuera, Manuel Orbegozo y José Manuel Corcuera.

Sin embargo, la obra de Efraín Orbegoso también adolece de serias omisiones, aparte de haber fijado su atención principalmente en maestros naturales de la capital de la provincia. Entonces, no incluye en su relación a destacados intelectuales, como el ensayista, poeta, periodista y jurista Víctor Julio Ortecho; el investigador educacional Alberto Moya Obeso, de notable producción intelectual; los poetas Juan Paredes Carbonell y Claudio Saya, ambos, esclarecidos miembros del Grupo "Trilce”, de Trujillo; y también, sobre todo el primero, de notable y prestigiosa producción literaria

\subsection{MAESTROS SANTIAGUinOS}

\section{CÉSAR VALLEJO ENTRE LA DOCENCIA Y LA ANÉCDOTA}

Si bien la trayectoria de César Vallejo está dominada por su excelencia poética y literaria, no menos importante es su función docente, que la inició a temprana edad, a los 19 años, en 1911, cuando es contratado como "preceptor" de los niños Francisco y Leoncio, hijos del señor Domingo Sotil, hacendado de Acobamba, provincia de Ambo, en Huánuco.

En 1913 busca trabajo con el propósito de solventar sus estudios universitarios y consigue una plaza como profesor del Centro Escolar de Varones $\mathrm{N}^{\circ}$ 241, ubicado en la Plaza de Armas de Trujillo, y designado actualmente como Institución Educativa $N^{\circ}$ 81014, "Pedro M. Ureña". Allí enseñó actividades relacionadas con las ciencias, el arte y las humanidades y para ilustrar mejor las lecciones para sus alumnos componía poesía didáctica que publicó en diversos 
números de "Cultura Infantil", revista pionera en el campo de la literatura infantil y juvenil peruana, fundada por el director de la escuela Julio Eduardo Mannucci. Entre otros poemas que publicó en dicha revista figuran: "Fosforescencia”, "Transpiración vegetal", "Fusión”, etc.

Como rasgo de su personalidad pedagógica Vallejo se distingue por una generosidad y comprensión ilimitadas hacia sus alumnos, signo de lo cual es la siguiente anécdota recogida por Juan Espejo Asturrizaga, que nosotros lo hemos publicado bajo el título "El alumno tardón y el maestro consentidor":

En el tercer año de primaria del Centro Escolar $N^{\circ} 241$, de Trujillo, también conocido como Centro Viejo, a cargo del profesor César Vallejo, cursaba estudios un alumno muy delgadito, muy fino, que siempre llegaba tarde a clases. De acuerdo con el reglamento de la escuela, a los alumnos que llegaban retrasados se les castigaba dejándolos como reclusos una hora todas las tardes, de pie, en un gran patio, hasta las seis de la tarde, ya que la hora de salida era a las cinco. Sin embargo, pese a la estrictez del reglamento, el pequeño alumno nunca era llamado cuando se leían las listas de reclusos, hecho que no dejaba de sorprenderlo; hasta que una mañana se esforzó en llegar temprano. Entonces, el profesor Vallejo se acercó a su alumnito y con mucho cariño, tomándole la cabecita, le dijo:

-Tú puedes llegar tarde siempre; no le diré a nadie. Eres muy flaquito y te hace daño agitarte.

Yacariciando más a su pequeño alumno, añadió:

-Me gusta tu cabeza...

Sin dejar de colaborar en la mencionada revista, en 1915 Vallejo pasó a trabajar en el Colegio Nacional de San Juan, nivel primario. En este colegio trabajó en los dos años siguientes, hasta 1917, cuando tuvo como alumno al futuro novelista Ciro Alegría, quien ha evocado a su ilustre maestro en la crónica testimonial "El César Vallejo que yo conocí” incluido en las Memorias "Mucha suerte con harto palo". De su paso por tan prestigioso colegio el mismo Espejo ha recogido la siguiente anécdota:

\section{¡SHIT!... EL POETA ESTÁ DURMIENDO}

El profesor César Vallejo enseñaba en el Colegio Nacional de San Juan, de Trujillo, a los niños que cursaban el primer año de educación primaria.

Por entonces, ante el retiro de la docencia del doctor Gustavo Ries, acababa de hacerse cargo de la dirección del colegio el doctor Juan de Dios Loray Cordero.

El nuevo director acostumbraba, tanto en las mañanas como en las tardes, después de iniciadas las labores, hacer un recorrido por los pasadizos y claustros del plantel, para comprobar la asistencia del personal docente. 
Una tarde, cumplida la primera hora, empezó su recorrido habitual. Era un caluroso día de verano. Avanzando por el claustro del segundo patio, donde funcionaba la sección primaria, le llamó la atención el gran silencio que reinaba en el aula del primer año que, por tratarse de niños -los más pequeños del plantel-, deberían estar, como siempre, movidos y bulliciosos, si es que el profesor no les dictaba clases. Se acercó, pues, el director a la puerta y, con gran sorpresa, contempló al profesor que era nada menos que el ya conocido por él, César Vallejo, quien, reclinado sobre su pupitre, dormía profundamente, mientras los niños en el salón permanecían en el más grave y respetuoso silencio. Ante la sorpresiva presencia del director, los niños trataron de ponerse de pie, pero, reaccionando de inmediato, el doctor Lora les hizo en forma apresurada señales con ambas manos para que no se movieran y, llevándose el índice a los labios: “;Shist!”, les invocó silencio.

Y prosiguió su paseo por los demás salones del claustro.

Sobre las materias que enseñaba, su estilo de enseñanza y la imagen que empezaba a proyectar Vallejo en la comunidad, su alumno Ciro Alegría lo recuerda así:

César Vallejo nos enseñaba rudimentos de historia, geografía, religión, matemáticas y a leer y escribir. También trataba de enseñarnos a cantar, pero nosotros lo hacíamos mejor que él, pues tenía muy mala voz. En cuanto a marchar, no se preocupaba de que lo hiciéramos bien, cosa en que ponían gran empeño con sus condiscípulos los maestros de grados superiores. Cuando los alumnos del colegio pasábamos en formación por las calles, yendo al campo de paseo o en los desfiles del 28 de julio, los del primer año de primaria, con nuestro melenudo profesor a la cabeza, no marcábamos regularmente el paso y éramos una tropilla bastante desgarbada. Oíamos que la gente estacionada en las aceras murmuraba viendo a nuestro profesor: “'Ahí va Vallejo! ¡Ahí va Vallejo!”.

Al concluir el último año de estudios de Jurisprudencia y la culminación del año escolar en el que se desempeñó como maestro, Vallejo viajó a la capital de la República en busca de nuevos horizontes. Al iniciarse el año escolar de 1918 fue contratado como profesor de primaria en uno de los mejores colegios particulares, el Colegio Barrós (Jr. Ancash, 506). Pero en setiembre del mismo año muere el director, fundador y propietario del colegio, el Dr. Pedro M. Barrós. La familia decide cancelar el funcionamiento del colegio, pero se permite que los docentes puedan continuar su tarea bajo su responsabilidad y otro nombre del colegio. Vallejo y algunos colegas llevan adelante la nueva institución bajo el nombre de "Instituto Nacional", ubicado en la calle Santa Clara N 910. Como director fue muy cuidadoso y responsable en la conducción del plantel, aparte de que tuvo a su cargo el desarrollo de las asignaturas de Castellano e Historia del Perú. 
En 1919 consigue puesto como maestro en el Colegio Nacional "Nuestra Señora de Guadalupe", siendo ese año director el Dr. Gustavo Ries, quien ya lo había contratado anteriormente en el Colegio San Juan, de Trujillo. En dicho colegio tuvo a su cargo sucesivamente las secciones de cuarto año con 41 alumnos y quinto, con 53 alumnos de primaria.

$\mathrm{Al}$ año siguiente, cuando el colegio tuvo como director al profesor Carlos Hilburg, se redujo la sección primaria y el poeta quedó en condición de cesante, circunstancia que determinó su regreso a Santiago de Chuco. No obstante, el mencionado director Hilburg decide nombrarlo como inspector de disciplina. Seguramente a esta época corresponde la siguiente anécdota:

\section{PAPAS A LA HUANCAÍNA}

El biógrafo de Vallejo, Juan Espejo Asturrizaga, refiere que al iniciarse las labores escolares César Vallejo, quien venía desempeñándose como profesor de Educación Primaria en el Colegio Nuestra Señora de Guadalupe, fue informado por el director de que, por razones económicas, se habian adoptado medidas de reducción de personal, por lo que se habian visto obligados a declararlo cesante. El profesor César Vallejo, lejos de abatirse o lamentarse, recibió la noticia con ese sentido del humor que empezaba ya a ser una característica definida de su personalidad cada vez que se enfrentaba a sucesos duros, aciagos y dolorosos. Por eso, cuando alguien le preguntó a qué se dedicaría en adelante, el profesor Vallejo respondió con toda tranquilidad:

-Me voy a sembrar papas a la huancaína.

\section{LoS HERMANOS PEREDA HidALGO}

En el prólogo al poemario "Machu Picchu" (2011), el poeta, narrador, ensayista y promotor cultural Danilo Sánchez Lihón nos ofrece la imagen y el perfil de dos notables maestros e intelectuales santiaguinos:

Era un clan, el de los Pereda Hidalgo, principalmente compuesto por Santiago, Inti Illapa, y don Julio, Inti Túpac, quienes reverenciaban al sol, lo saludaban cada mañana en el patio de su casa, hacían el ritual sagrado de venerar a la Pacha Mama, a la vida, a la luz, a la energía viviente del cosmos. Don Santiago aprendió a hablar el quechua y lo practicaba en la intimidad, porque en Santiago de Chuco lamentablemente no se habla el quechua de manera corriente.

Nació Santiago Pereda Hidalgo el 10 de agosto del año 1900 en el lugar denominado Punchaypampa, arriendo ubicado en una zona aledaña a Calipuy, en la provincia de Santiago de Chuco. 
A los 4 años ya sabía leer y escribir. Realizó sus estudios primarios en Santiago de Chuco y los secundarios en el Instituto Moderno de Trujillo, dirigido por los hermanos Carlos y Santiago Uceda, notables maestros nacidos y criados en Santiago de Chuco.

En esa institución educativa fue un alumno brillante desde el primer día de clases. Obtuvo calificaciones de excelencia, participó en diversos actos cívicos. Inmediatamente después de graduarse con las más altas notas ingresó a trabajar como profesor en ese mismo centro de estudios. Integró la banda y el coro, aprendió a tocar la guitarra y el violín, y pronto se le confió la dirección del nivel primario. En la revista quincenal "Ensayo", del mismo Instituto, publicó su primer poema, "El Peregrino", en el año 1919.

Regresó a Santiago para ejercer su profesión en el distrito de Chuca, donde fundó y dirigió su propia escuela, a la que denominó Yachay Huasi ("Escuela del amor"), proyecto educativo con identidad propia que desarrolló conjuntamente con su hermano Julio, cuyo seudónimo literario era Inti Túpac, quien editó para ese proyecto bellos y primorosos libros para niños.

El primer libro de lectura de Inti Túpac o Julio Pereda (n. en Santiago de Chuco en 1904), se tituló Canta, pajarillo... También publicó “Inti Raymi” y "Canto de Navidad".

Con Santiago y sus demás hermanos crearon la Editorial Inti, donde se publicaron algunas obras, el periódico "Huanacaure" y se brindó diversos servicios a la ciudad.

Posteriormente, Santiago Pereda Hidalgo, por sus propios méritos profesionales, ocupó el cargo de Inspector de Educación, equivalente al cargo actual de supervisor de educación, gestión en la cual puso en ejecución el anhelado proyecto de las escuelas comunales, varias de las cuales funcionaron en el ámbito rural, dotadas de sus respectivas planas docentes, locales escolares, material educativo, recursos didácticos adecuados y, sobre todo, con una visión propia de la escuela en función de su entorno vital.

La experiencia realizada por primera vez en Santiago de Chuco resultó tan exitosa, que el Ministerio de Educación quiso que se replicara y se extendiera por el ser del país, por lo que el maestro santiaguino fue nombrado autoridad educativa en Acobamba, Huancavelica.

La obra de los hermanos Santiago y Julio resultó extraordinaria. En todo pueblo donde trabajaron alentaron inquietudes y anhelos. No sucumbieron a la inercia, ni al apartamiento, ni a la marginalidad. Escribían en cada pueblo, per- 
filaban materiales en cada aldea, innovaban concepciones desde cada paraje o rincón, hacienda, caserío o poblado.

Son obras publicadas por Santiago Pereda Hidalgo: Koricancha, poemas (1948), Aklla, poemas (s/f), Imanacha tukukun. El fin del Tahuantinsuyo, tomo I, 1946, reconstrucción histórica novelada del Imperio de los Incas, su grandeza y su fin, La revolución peruana y la libertad del pueblo de los Incas (1969), Machu Picchu (1974), Canto de revolución a Túpac Amaru, Paz o Destrucción, novela acerca del indio peruano.

A los hermanos Pereda Hidalgo se los silenciaba en el momento en que aparecían sus libros. Pero no solo es este un alejamiento de las personas, sino que es una marginación a una cultura, a un mundo como es el andino y a una manera de ser, de sentir, de pensar y de actuar que consuetudinariamente se lo relega en nuestro país, como es el legado de nuestros antepasados y que sin embargo es lo mejor que tenemos.

\section{ABRAHAM ARIAS LARRETA}

Egresado muy joven de la Escuela Normal de Varones de Lima, empezó a ejercer el magisterio en la ciudad de Tacna. Su vocación de maestro la alternó con su pasión por la literatura y el periodismo. En dicha ciudad fundó la revista "Perú".

Fue nombrado Director de llamada "Escuela de Abajo" y luego Inspector de Educación en la ciudad de Huaraz (1930). Posteriormente fue trasladado a Chiclayo y después a Lima. Por sus ideas y militancia política en el Partido Aprista fue deportado a Argentina, de donde pasó a Chile, en que visitó a Gabriela Mistral hasta en dos oportunidades. De dicha visita ella le envió una hermosa carta que se incluyó a manera de prólogo en la primera edición de "Rayuelo".

Después de los cruentos años de la revolución de Trujillo (1932) se dedicó a la docencia, a las tareas administrativas y a la creación literaria. También tomó parte activa en la formación de las primeras organizaciones del magisterio. Precisamente, a raíz de un artículo publicado en "El Comercio" acerca de los bajos sueldos de los maestros, fue separado de la docencia y pasó a laborar en colegios particulares; sin desmayar en sus afanes reivindicacionistas, contribuyó a la formación de la Asociación de Maestros de Lima y Balnearios. El despliegue de sus actividades determinó que fuera vigilado, acosado, perseguido y preso en la colonia penal "El Frontón”, entre los años 1939-43. Una vez en libertad retornó a Trujillo, donde se desempeñó como secretario privado de don 
Rafael Larco Herrera en la hacienda Chiclín, al mismo tiempo que se dedica a escribir e investigar.

Entre 1945-47 se desempeñó como catedrático de Literatura Peruana y Latinoamericana en la Universidad Nacional de Trujillo. Tal situación le permitió desarrollar y difundir su tesis sobre las literaturas aborígenes y la insurgencia provinciana. Precisamente por esos años (1947) publicó sus ensayos "Radiografía de la literatura peruana" y "Folklore Nor Peruano". También fundó la importante revista "Sayari" de literatura, polémica y doctrina. Al producirse ciertas pugnas en el interior de su partido fue incluido entre los desobedientes a las cúpulas dirigenciales y se le suspendió por seis meses.

Casi simultáneamente pasó a trabajar como Profesor Visitante en las universidades de Caracas, Guatemala y México (1948) y a continuación fue designado catedrático de Español y Literatura Hispanoamericana en la Universidad de California, Los Angeles, (1949). A partir de allí ejerció la docencia en las universidades de: Stanford, Washington, Seattle, Missisipi, State University y Missouri. En Norteamérica publicó gran parte de sus obras, principalmente sobre didáctica y tratados de literatura.

Falleció el 24 de octubre de 1980 en Kansas City, Missouri.

\section{DANILO SÁNCHEZ GAMBOA Y CARLOS BARBARÁN}

Danilo Sánchez Lihón, de indiscutible, indesmayable y fervoroso amor por su tierra y sus valores humanos es autor también de dos formidables y estupendos libros destinados a destacar la memoria y la obra de dos insignes maestros santiaguinos: "Alma de maestro" (2002) y "Vida y obra de un maestro: Carlos Barbarán” (2003). Además de la brillante prosa que caracteriza al autor, ambos libros desbordan cariño, devoción, gratitud y reconocimiento por la extraordinaria labor pedagógica que cumplieron los referentes humanos que son el motivo inspirador de ambos volúmenes. Pero la trayectoria intelectual de Danilo no se limita a la publicación de las mencionadas obras. Su producción por el desarrollo y promoción de la literatura infantil, la animación lectora, la defensa y propagación del folclore, el estímulo al desarrollo de la creatividad, etc. se han plasmado en una densa, vasta y diversa obra, en cuyo conjunto podemos citar, sólo a manera de ilustración, los títulos: "Encinas, maestro del Perú”, "Cómo leer mejor”, "Te regalo el arcoiris”, "Lectura y textos escolares", "La narración de cuentos y su función en la educación", "Proceso de la literatura infantil en el Perú", "Literatura infantil, magia y realidad”, "El niño 
y el mundo por crear”, etc. En esta oportunidad, de acuerdo a la naturaleza de la presente obra, nos centramos en los títulos indicados en primer término.

"Alma de maestro" es también un hermoso y sublime tributo de amor filial en el que el autor explora, reconstruye, evoca y valora la importancia pedagógica de su padre Danilo Sánchez Gamboa:

De allí que esta obra no deje de ser un libro testimonial y en buena cuenta, de memorias y, como tal, de vivencias en relación con un personaje muy cercano a la vida de su autor. En tal sentido, en algunos o en frecuentes momentos, no parecerá una obra que se ubique en el campo de la educación, siendo ése nuestro postulado y propósito, cual es que la educación se parezca cada vez más a la vida y que se identifique más íntegramente con ella.

Por eso está escrito desde lo emocional y desde experiencias ligadas a la vida y a la mirada de un niño. Tanto que pareciera ser un libro de recuerdos personales, pero que en el fondo y en su esencia no se aparta del sentido pedagógico que persigue, de la misión educativa que intento cumplir, cual encontrar y significar la educación en los hechos cotidianos que marcan a las personas y que, en este caso, se testimonia en quienes más próximos estuvimos a aquel maestro ( $p$. 18).

En otros párrafos de su extensa presentación, Danilo aclara que es verdad que el protagonista de su libro es su propio padre, circunstancia por la que siente auténtico y legítimo orgullo, pero que es vida trascendente es también equiparable a la de tantos maestros del Perú, por lo que justifica concentrarse y llamar la atención en estos puntos:

Siento, es cierto, orgullo de un hombre que se lavaba él mismo su propia ropa, se cocinaba solo, se remendaba los vestidos pese a su valía y que tocaba la mandolina en una sala y en un patio desolados; sólo por la fuerza de amar y vivir en el ámbito de los ideales y de los sueños; que aspiraba y ponía su brazo-terca y obstinadamente- a favor de un porvenir de grandes realizaciones para su patria, el Perú (p. 19).

A continuación, el autor recrea los pasos y trayectoria del maestro a través del registro minucioso de sus actividades en la escuela, la participación musical en los ensayos y en las actuaciones extraordinarias y singulares que rompen la tranquilidad cotidiana del pueblo para traspasarse de armonía sutil y superior. Recrea determinadas estampas de los escolares y maestros en épocas propicias, como el mes de las cometas, las intervenciones deportivas y la rutina feliz y nostálgica de los fines de año, así como exalta también los múltiples actos de desprendimiento que tuvo el maestro siempre y más allá de las aulas, en el propio pueblo o en la gran ciudad. 
El otro libro de similar orientación en cuanto a exaltar las virtudes y trayectoria de un hombre consagrado a la formación de niños y jóvenes es "Vida y obra de un maestro Carlos Barbarán”, cuyas páginas están destinadas, en su primera parte, a exaltar la singular personalidad y labor pedagógica, cultural, folclórica, del inspirador de la obra, con participación directa en actividades sociales, científicas, pedagógicas, artísticas y gremiales, pues con su intervención, la de su colega Romeo Solís Rosas y la adhesión de Andrés Camino Carranza, hijo del escritor Carlos Camino Calderón, lograron, por fin, la creación del Colegio Particular Santiago El Mayor. También por sus gestiones es que se fundó el Sindicato de Maestros de la provincia de Santiago de Chuco, el primero en crearse en el Perú, en 1957, en base a la conversión de la ya existente Asociación de Maestros; posteriormente surgiría el del Sur Chico (Cañete, Chincha e Ica). Entonces, la personalidad y la imagen del maestro, las reflejan estas líneas:

Destacábase en él sobre todo el imperio y la égida del ideal. Abominaba lo mediocre y relajado, lo vulgar y lo chabacano. Sin embargo, lo más frecuente era su sonrisa radiante y bondadosa, su luminosidad en el vestir y en el gesto, su talante vital en la dimensión del espíritu. Con mucha armonía interior, perspicaz para tomar nota de lo que acontecía a su alrededor. Muy exigente en la conducta, en el buen comportamiento dentro del Colegio como con lo que se desenvolvía en la calle y en el ámbito del pueblo en relación con los alumnos.

A veces, de pie, en el corredor tenía el rostro contraído con el libro entre sus manos mientras nosotros jugábamos o conversábamos en el patio alumbrado por el sol de nuestro cielo. Era un profesor severo, temido por su rectitud y ante quien bastaba una brizna fuera de su sitio para que se le encendiera el rostro, chispearan sus ojos fulgurantes y ordenara a los elementos terráqueos que estallen. Sus cóleras eran temibles, pero pasajeras. Pronto sonreía con recogimiento y con dulzura dejando ver una dentadura pareja y armoniosa.

Jamás lo vi de juerga, en una cantina o en una actitud displicente. Reaccionaba indignado ante cualquier desparpajo o actitud equívoca, a veces mínima. Un alumno en el salón le encendió de cólera porque masticaba un palito de fósforo que lo hacía pender de un costado de la boca. No era complaciente con el error. Todo lo quería perfecto y el primero en ser exigido era él mismo (p. 32).

La parte central del libro, sin embargo está destinada a reproducir las partes medulares de dos tesis: la primera, "La realidad socio-escolar de Santiago de Chuco", con la que optó el título de Profesor de Segunda Enseñanza, presentada en la Facultad de Educación de la Pontificia Universidad Católica del Perú, en 1959. Su otra tesis, "Ensayo monográfico de la provincia de Santiago de Chuco", le permitió optar el Grado Académico de Bachiller en Huma- 
nidades en la Universidad Nacional de Trujillo. Ambas obras contienen aspectos de notable valor para el conocimiento y valoración de la respectiva provincia. Tal importancia se puede vislumbrar por el registro de los principales temas: Antecedentes históricos de Santiago de Chuco, la realidad socio-escolar, la condición del maestro; asimismo, aspectos como la nutrición de la población, la ocupación laboral, las actividades festivas, cuentos y tradiciones, supersticiones y creencias, costumbres, cancionero popular y coplas; asimismo estudios sobre el clima, la flora y la fauna, orografía, hidrografía y mineralogía; del mismo modo, el valor se agranda por la inclusión de temas folclóricos nativos (el Quishpi cóndor, las quiyayas), aspectos lingüísticos y el relato "El Juan Osito", versión propia del norte del Perú.

La mencionada obra fue editada como parte del homenaje por el centenario de la fundación de la provincia de Santiago de Chuco (2000).

\section{REFERENCIAS BIBLIOGRÁFICAS}

ALEGRíA, Ciro. Memorias: mucha suerte con harto palo. Lima, Ediciones Varona, 1978.

CASTILLO SÁNChEZ, WeLLington. Los hermanos Arias Larreta: Identidad y compromiso. Trujillo, Editorial La Razón, 1995.

GALARRETA GONZALEZ, JULIO. Abelardo Gamarra, pensador peruano. Lima, PL Villanueva Talleres Gráficos, 1974.

GAMARRA GonZALEZ, Julio. Abelardo Gamarra, educador peruano. Lima, Universidad Nacional "Federico Villarreal", 1974.

Moya Obeso, Alberto Santiago. Pensar/investigar en la Era del conocimiento. Lima, Editorial San Marcos, 2010.

ORbEgoso RodríGueZ, EFraín. Cuatro ensayos sobre la Región La Libertad. Lima, Pie de Trigo Editores \& Publicistas, 2000.

SÁNCHEZ LiHón, DANILO. Alma de maestro. Lima, Instituto del Libro y la Lectura (INLEC), 2002.

SÁNCHEZ LiHÓN, DANILO. Vida y obra de un maestro: Carlos Barbarán. Lima, Instituto del Libro y la Lectura (INLEC), 2003).

SÁNCHEZ LiHÓn, DANILO. Prólogo a Machu Picchu de Inti Illapa. Lima, Editorial Pasacalle, 2011. 